\title{
Preliminaries to Classical First Order Model Theory ${ }^{1}$
}

\author{
Marco B. Caminati ${ }^{2}$ \\ Mathematics Department "G.Castelnuovo" \\ Sapienza University of Rome \\ Piazzale Aldo Moro 5, 00185 Roma, Italy
}

\begin{abstract}
Summary. First of a series of articles laying down the bases for classical first order model theory. These articles introduce a framework for treating arbitrary languages with equality. This framework is kept as generic and modular as possible: both the language and the derivation rule are introduced as a type, rather than a fixed functor; definitions and results regarding syntax, semantics, interpretations and sequent derivation rules, respectively, are confined to separate articles, to mark out the hierarchy of dependences among different definitions
\end{abstract} and constructions.

As an application limited to countable languages, satisfiability theorem and a full version of the Gödel completeness theorem are delivered, with respect to a fixed, remarkably thrifty, set of correct rules. Besides the self-referential significance for the Mizar project itself of those theorems being formalized with respect to a generic, equality-furnished, countable language, this is the first step to work out other milestones of model theory, such as Lowenheim-Skolem and compactness theorems. Being the receptacle of all results of broader scope stemmed during the various formalizations, this first article stays at a very generic level, with results and registrations about objects already in the Mizar Mathematical Library.

Without introducing the Language structure yet, three fundamental definitions of wide applicability are also given: the 'unambiguous' attribute (see [20], definition on page 5), the functor '-multiCat', which is the iteration of " a FinSequence of FinSequence, and the functor SubstWith, which realizes the substitution of a single symbol inside a generic FinSequence.

MML identifier: FOMODELO, version: $\underline{7.11 .07 \quad 4.160 .1126}$

\footnotetext{
${ }^{1}$ The author wrote this paper as part of his $\mathrm{PhD}$ thesis research.

${ }^{2}$ I would like to thank Marco Pedicini for his encouragement and support.
} 
The papers [11], [2], [4], [12], [23], [7], [13], [19], [22], [14], [15], [10], [16], [9], [25], [1], [27], [8], [24], [6], [3], [5], [17], [28], [30], [29], [21], [26], and [18] provide the notation and terminology for this paper.

For simplicity, we adopt the following rules: $U, D$ are non empty sets, $X$ is a non empty subset of $D, d$ is an element of $D, A, B, C, Y, x, y, z$ are sets, $f$ is a binary operation on $D, i, m, n$ are natural numbers, and $g$ is a function.

Let $X$ be a set and let $f$ be a function. We say that $f$ is $X$-one-to-one if and only if:

(Def. 1) $f\lceil X$ is one-to-one.

Let us consider $D, f$ and let $X$ be a set. We say that $X$ is $f$-unambiguous if and only if:

(Def. 2) $f$ is $X \times D$-one-to-one.

Let us consider $D$ and let $X$ be a set. We say that $X$ is $D$-prefix if and only if:

(Def. 3) $X$ is (the concatenation of $D$ )-unambiguous.

Let $D$ be a set. The functor $D$-pr1 yielding a binary operation on $D$ is defined by:

(Def. 4) $\quad D$-pr1 $=\pi_{1}(D \times D)$.

One can prove the following propositions:

(1) $A^{m} \cap B^{*}=A^{m} \cap B^{m}$.

(2) $A^{m} \cap B^{*}=(A \cap B)^{m}$.

(3) $(A \cap B)^{m}=A^{m} \cap B^{m}$.

(4) For all finite sequences $x, y$ such that $x$ is $U$-valued and $y$ is $U$-valued holds (the concatenation of $U)(x, y)=x^{\frown} y$.

(5) For every set $x$ holds $x$ is a non empty finite sequence of elements of $D$ iff $x \in D^{*} \backslash\{\emptyset\}$.

Let $D$ be a non empty set. One can check that $D$-pr1 is associative.

Let $D$ be a set. Note that there exists a binary operation on $D$ which is associative.

Let $X$ be a set and let $Y$ be a subset of $X$. Then $Y^{*}$ is a non empty subset of $X^{*}$.

Let $D$ be a non empty set. Observe that the concatenation of $D$ is associative. Observe that $D^{*} \backslash\{\emptyset\}$ is non empty.

Let $m$ be a natural number. Note that there exists an element of $D^{*}$ which is $m$-element.

Let $X$ be a set and let $f$ be a function. Let us observe that $f$ is $X$-one-to-one if and only if:

(Def. 5) For all sets $x, y$ such that $x, y \in X \cap \operatorname{dom} f$ and $f(x)=f(y)$ holds $x=y$.

Let us consider $D, f$. Note that there exists a set which is $f$-unambiguous. 
Let $f$ be a function and let $x$ be a set. Note that $f\lceil\{x\}$ is one-to-one.

One can verify that every set which is empty is also empty-membered. Let $e$ be an empty set. Note that $\{e\}$ is empty-membered.

Let us consider $U$ and let $m_{1}$ be a non zero natural number. Observe that $U^{m_{1}}$ has non empty elements.

Let $X$ be an empty-membered set. Note that every subset of $X$ is emptymembered.

Let us consider $A$ and let $m_{0}$ be a zero number. Note that $A^{m_{0}}$ is emptymembered.

Let $e$ be an empty set and let $m_{1}$ be a non zero natural number. Observe that $e^{m_{1}}$ is empty.

Let us consider $D, f$ and let $e$ be an empty set. One can verify that $e \cap f$ is $f$-unambiguous.

Let us consider $U$ and let $e$ be an empty set. One can check that $e \cap U$ is $U$-prefix.

Let us consider $U$. Observe that there exists a set which is $U$-prefix.

Let us consider $D, f$ and let $x$ be a finite sequence of elements of $D$. The functor MultPlace $(f, x)$ yields a function and is defined by:

(Def. 6) $\operatorname{dom} \operatorname{MultPlace}(f, x)=\mathbb{N}$ and $(\operatorname{MultPlace}(f, x))(0)=x(1)$ and for every natural number $n$ holds $($ MultPlace $(f, x))(n+1)=$ $f((\operatorname{MultPlace}(f, x))(n), x(n+2))$.

Let us consider $D, f$ and let $x$ be an element of $D^{*} \backslash\{\emptyset\}$. The functor MultPlace $(f, x)$ yields a function and is defined as follows:

(Def. 7) $\operatorname{MultPlace}(f, x)=\operatorname{MultPlace}\left(f,\left(x\right.\right.$ qua element of $\left.\left.D^{*}\right)\right)$.

Let us consider $D, f$. The functor MultPlace $f$ yielding a function from $D^{*} \backslash\{\emptyset\}$ into $D$ is defined as follows:

(Def. 8) For every element $x$ of $D^{*} \backslash\{\emptyset\}$ holds $($ MultPlace $f)(x)=$ (MultPlace $(f, x))($ len $x-1)$.

Let us consider $D, f$ and let $X$ be a set. Let us observe that $X$ is $f$ unambiguous if and only if:

(Def. 9) For all sets $x, y, d_{1}, d_{2}$ such that $x, y \in X \cap D$ and $d_{1}, d_{2} \in D$ and $f(x$, $\left.d_{1}\right)=f\left(y, d_{2}\right)$ holds $x=y$ and $d_{1}=d_{2}$.

Let us consider $D$. The functor $D$-firstChar yields a function from $D^{*} \backslash\{\emptyset\}$ into $D$ and is defined as follows:

(Def. 10) $D$-firstChar $=\operatorname{MultPlace}(D$-pr1).

One can prove the following proposition

(6) For every finite sequence $p$ such that $p$ is $U$-valued and non empty holds $U$-firstChar $(p)=p(1)$.

Let us consider $D$. The functor $D$-multiCat yielding a function is defined as follows: 
(Def. 11) $D$-multiCat $=(\emptyset \longmapsto \emptyset)+\cdot$ MultPlace (the concatenation of $D)$.

Let us consider $D$. Then $D$-multiCat is a function from $\left(D^{*}\right)^{*}$ into $D^{*}$.

Let us consider $D$ and let $e$ be an empty set. One can check that $D$-multiCat $(e)$ is empty.

Let us consider $D$. Observe that every subset of $D^{1}$ is $D$-prefix.

The following propositions are true:

(7) If $A$ is $D$-prefix, then $D$-multiCat ${ }^{\circ} A^{m}$ is $D$-prefix.

(8) If $A$ is $D$-prefix, then $D$-multiCat is $A^{m}$-one-to-one.

(9) $Y^{m+1} \subseteq Y^{*} \backslash\{\emptyset\}$.

(10) If $m$ is zero, then $Y^{m}=\{\emptyset\}$.

(11) $Y^{i}=Y^{\operatorname{Seg} i}$.

(12) If $x \in A^{m}$, then $x$ is a finite sequence of elements of $A$.

Let $A, X$ be sets. Then $\chi_{A, X}$ is a function from $X$ into Boolean.

Next we state three propositions:

(13) (MultPlace $f)(\langle d\rangle)=d$ and for every non empty finite sequence $x$ of elements of $D$ holds (MultPlace $f)\left(x^{\frown}\langle d\rangle\right)=f(($ MultPlace $f)(x), d)$.

(14) For every non empty element $d$ of $\left(D^{*}\right)^{*}$ holds $D$-multiCat $(d)=$ (MultPlace (the concatenation of $D)$ ) $(d)$.

(15) For all elements $d_{1}, d_{2}$ of $D^{*}$ holds $D$-multiCat $\left(\left\langle d_{1}, d_{2}\right\rangle\right)=d_{1} \frown d_{2}$.

Let $f, g$ be finite sequences. One can verify that $\langle f, g\rangle$ is finite sequence-like.

Let us consider $m$ and let $f, g$ be $m$-element finite sequences. Note that $\langle f, g\rangle$ is $m$-element.

Let $X, Y$ be sets, let $f$ be an $X$-defined function, and let $g$ be a $Y$-defined function. Observe that $\langle f, g\rangle$ is $X \cap Y$-defined.

Let $X$ be a set and let $f, g$ be $X$-defined functions. Observe that $\langle f, g\rangle$ is $X$-defined.

Let $X, Y$ be sets, let $f$ be a total $X$-defined function, and let $g$ be a total $Y$-defined function. Note that $\langle f, g\rangle$ is total.

Let $X$ be a set and let $f, g$ be total $X$-defined functions. Note that $\langle f, g\rangle$ is total.

Let $X, Y$ be sets, let $f$ be an $X$-valued function, and let $g$ be a $Y$-valued function. One can verify that $\langle f, g\rangle$ is $X \times Y$-valued.

Let us consider $D$. Observe that there exists a finite sequence which is $D$ valued.

Let us consider $D, m$. Note that there exists a $D$-valued finite sequence which is $m$-element.

Let $X, Y$ be non empty sets, let $f$ be a function from $X$ into $Y$, and let $p$ be an $X$-valued finite sequence. Observe that $f \cdot p$ is finite sequence-like.

Let us consider $m$, let $f$ be a function from $X$ into $Y$, and let $p$ be an $m$-element $X$-valued finite sequence. Note that $f \cdot p$ is $m$-element. 
Let us consider $D, f$ and let $p, q$ be elements of $D^{*}$.

The functor $f$ AppliedPairwiseTo $(p, q)$ yields a finite sequence of elements of $D$ and is defined by:

(Def. 12) $f$ AppliedPairwiseTo $(p, q)=f \cdot\langle p, q\rangle$.

Let us consider $D, f, m$ and let $p, q$ be $m$-element elements of $D^{*}$. Note that $f$ AppliedPairwiseTo $(p, q)$ is $m$-element.

Let us consider $D, f$ and let $p, q$ be elements of $D^{*}$. We introduce $f \backslash(p, q)$ as a synonym of $f$ AppliedPairwiseTo $(p, q)$.

$\mathbb{Z}$ can be characterized by the condition:

(Def. 13) $\mathbb{Z}=\mathbb{N} \cup(\{0\} \times \mathbb{N} \backslash\{\langle 0,0\rangle\})$.

We now state the proposition

(16) For every finite sequence $p$ such that $p$ is $Y$-valued and $m$-element holds $p \in Y^{m}$.

Let us consider $A, B$. The functor $A \leftarrow \cap B$ yields a subset of $A$ and is defined by:

(Def. 14) $\quad A \leftarrow \cap B=A \cap B$.

The functor $A \cap \rightarrow B$ yielding a subset of $B$ is defined as follows:

(Def. 15) $\quad A \cap \rightarrow B=A \cap B$.

Let us consider $B, A$. The functor $A$ null $B$ is defined by:

(Def. 16) $A$ null $B=A$.

Let us consider $A, B, C$. One can check that $(B \backslash A) \cap(A \cap C)$ is empty.

Let us consider $A, B$. The functor $A \backslash \leftarrow B$ yields a subset of $A$ and is defined as follows:

(Def. 17) $A \backslash \leftarrow B=A \backslash B$.

Let us consider $A, B$. The functor $A \cup \leftrightarrow B$ yielding a subset of $A \cup B$ is defined by:

(Def. 18) $\quad A \cup \leftrightarrow B=A$.

For simplicity, we adopt the following convention: $X$ is a set, $P, Q, R$ are binary relations, $f$ is a function, $p, q$ are finite sequences, and $U_{1}, U_{2}$ are non empty sets.

Let $R$ be a binary relation. Note that $R^{*}$ is transitive and $R^{*}$ is reflexive.

The function plus from $\mathbb{C}$ into $\mathbb{C}$ is defined as follows:

(Def. 19) For every complex number $z$ holds plus $(z)=z+1$.

The following two propositions are true:

(17) If $\operatorname{rng} f \subseteq \operatorname{dom} f$, then $f^{*}=\bigcup\left\{f^{m_{2}}: m_{2}\right.$ ranges over elements of $\left.\mathbb{N}\right\}$.

(18) If $f \subseteq g$, then $f^{m} \subseteq g^{m}$.

Let $X$ be a functional set. Note that $\bigcup X$ is relation-like.

Next we state the proposition

(19) If $Y \subseteq B^{A}$, then $\bigcup Y \subseteq A \times B$. 
Let us consider $Y$. Observe that $Y \backslash Y$ is empty.

Let us consider $D, d$. One can check that $\left\{\operatorname{id}_{D}(d)\right\} \backslash\{d\}$ is empty.

One can prove the following propositions:

(20) $f=\{\langle x, f(x)\rangle ; x$ ranges over elements of $\operatorname{dom} f: x \in \operatorname{dom} f\}$.

(21) For every total $Y$-defined binary relation $R$ holds $\operatorname{id}_{Y} \subseteq R \cdot R^{\smile}$.

(22) $\quad D^{m+n}=(\text { the concatenation of } D)^{\circ}\left(D^{m} \times D^{n}\right)$.

(23) For all binary relations $P, Q$ holds $(P \cup Q)^{-1}(Y)=P^{-1}(Y) \cup Q^{-1}(Y)$.

(24) $\left(\chi_{A, B}\right)^{-1}(\{0\})=B \backslash A$ and $\left(\chi_{A, B}\right)^{-1}(\{1\})=A \cap B$.

(25) For every non empty set $y$ holds $y=f(x)$ iff $x \in f^{-1}(\{y\})$.

(26) If $f$ is $Y$-valued and finite sequence-like, then $f$ is a finite sequence of elements of $Y$.

Let us consider $Y$ and let $X$ be a subset of $Y$. Observe that every binary relation which is $X$-valued is also $Y$-valued.

Let us consider $A, U$. One can verify that every relation between $A$ and $U$ which is quasi total is also total.

The following propositions are true:

(27) Let $Q$ be a quasi total relation between $B$ and $U_{1}, R$ be a quasi total relation between $B$ and $U_{2}$, and $P$ be a relation between $A$ and $B$. If $P \cdot Q \cdot Q^{\smile} \cdot R$ is function-like, then $P \cdot Q \cdot Q^{\smile} \cdot R=P \cdot R$.

(28) For all finite sequences $p, q$ such that $p$ is non empty holds $\left(p^{\frown} q\right)(1)=$ $p(1)$.

Let us consider $U$ and let $p, q$ be $U$-valued finite sequences. One can check that $p^{\frown} q$ is $U$-valued.

Let $X$ be a set. We see that the finite sequence of elements of $X$ is an element of $X^{*}$.

Let us consider $U, X$. Let us observe that $X$ is $U$-prefix if and only if:

(Def. 20) For all $U$-valued finite sequences $p_{1}, q_{1}, p_{2}, q_{2}$ such that $p_{1}, p_{2} \in X$ and $p_{1} \frown q_{1}=p_{2} \frown q_{2}$ holds $p_{1}=p_{2}$ and $q_{1}=q_{2}$.

Let $X$ be a set. Observe that every element of $X^{*}$ is $X$-valued.

Let us consider $U, m$ and let $X$ be a $U$-prefix set. Observe that $U$-multiCat ${ }^{\circ} X^{m}$ is $U$-prefix.

Next we state the proposition

(29) $X \dot{-} Y=\emptyset$ iff $X=Y$.

Let us consider $x$. Note that $\operatorname{id}_{\{x\}} \dot{-}\{\langle x, x\rangle\}$ is empty.

Let us consider $x, y$. Observe that $(x \longmapsto y) \doteq\{\langle x, y\rangle\}$ is empty.

Let us consider $x$. Note that $\operatorname{id}_{\{x\}} \dot{-}(x \longmapsto x)$ is empty.

The following proposition is true

(30) $x \in D^{*} \backslash\{\emptyset\}$ iff $x$ is a $D$-valued finite sequence and non empty.

In the sequel $f$ denotes a binary operation on $D$. 
The following proposition is true

(31) (MultPlace $f)(\langle d\rangle)=d$ and for every $D$-valued finite sequence $x$ such that $x$ is non empty holds (MultPlace $f)\left(x^{\frown}\langle d\rangle\right)=f(($ MultPlace $f)(x)$, $d)$.

For simplicity, we adopt the following rules: $A, B, C, X, Y, Z, x, x_{1}, y, y_{1}$, $y_{2}$ are sets, $U, U_{1}, U_{2}, U_{3}$ are non empty sets, $u, u_{1}, u_{2}$ are elements of $U, P$, $R$ are binary relations, $f, g$ are functions, $k, m, n$ are natural numbers, $k_{1}, m_{2}$, $n_{1}$ are elements of $\mathbb{N}, m_{1}, n_{2}$ are non zero natural numbers, $p, p_{1}, p_{2}$ are finite sequences, and $q, q_{1}, q_{2}$ are $U$-valued finite sequences.

Let us consider $p, x, y$. Note that $p \widetilde{+}(x, y)$ is finite sequence-like.

Let us consider $x, y, p$. The functor $(x, y)$-SymbolSubstIn $p$ yielding a finite sequence is defined by:

(Def. 21) $\quad(x, y)$-SymbolSubstIn $p=p \widetilde{+}(x, y)$.

Let us consider $x, y, m$ and let $p$ be an $m$-element finite sequence. Observe that $(x, y)$-SymbolSubstIn $p$ is $m$-element.

Let us consider $X$. Observe that there exists a finite sequence which is $X$ valued.

Let us consider $x, U, u$ and let $p$ be a $U$-valued finite sequence. Observe that $(x, u)$-SymbolSubstIn $p$ is $U$-valued.

Let us consider $X, x, y$ and let $p$ be an $X$-valued finite sequence. Then $(x, y)$-SymbolSubstIn $p$ can be characterized by the condition:

(Def. 22) $\quad(x, y)$-SymbolSubstIn $p=\left(\operatorname{id}_{X}+\cdot(x, y)\right) \cdot p$.

Let us consider $U, x, u, q$. Then $(x, u)$-SymbolSubstIn $q$ is a finite sequence of elements of $U$.

Let us consider $U, x, u$. The functor $x$ SubstWith $u$ yielding a function from $U^{*}$ into $U^{*}$ is defined as follows:

(Def. 23) For every $q$ holds $(x \operatorname{SubstWith} u)(q)=(x, u)$-SymbolSubstIn $q$.

Let us consider $U, x, u$. Note that $x$ SubstWith $u$ is finite sequence-yielding.

Let $F$ be a finite sequence-yielding function and let $x$ be a set. Observe that $F(x)$ is finite sequence-like.

Let us consider $U, x, u, m$ and let $p$ be a $U$-valued $m$-element finite sequence. Note that $(x \operatorname{SubstWith} u)(p)$ is $m$-element.

Let $e$ be an empty set. One can verify that $(x \operatorname{SubstWith} u)(e)$ is empty.

Let us consider $U$. Note that $U$-multiCat is finite sequence-yielding.

One can verify that there exists a $U$-valued finite sequence which is non empty.

Let us consider $U, m_{1}, n$ and let $p$ be an $m_{1}+n$-element $U$-valued finite sequence. Observe that $\left\{p\left(m_{1}\right)\right\} \backslash U$ is empty.

Let us consider $U, m, n$ and let $p$ be an $m+1+n$-element element of $U^{*}$. One can check that $\{p(m+1)\} \backslash U$ is empty.

Let us consider $x$. Note that $\langle x\rangle-\{\langle 1, x\rangle\}$ is empty. 
Let us consider $m$ and let $p$ be an $m+1$-element finite sequence. Observe that $\left(p\lceil\operatorname{Seg} m)^{\frown}\langle p(m+1)\rangle \dot{-} p\right.$ is empty.

Let us consider $m, n$ and let $p$ be an $m+n$-element finite sequence. One can verify that $p\lceil\operatorname{Seg} m$ is $m$-element.

Let us observe that every binary relation which is $\{\emptyset\}$-valued is also empty yielding and every binary relation which is empty yielding is also $\{\emptyset\}$-valued.

The following two propositions are true:

(32) $U$-multiCat $(x)=($ MultPlace $($ the concatenation of $U))(x)$.

(33) If $p$ is $U^{*}$-valued, then $U$-multiCat $\left(p^{\frown}\langle q\rangle\right)=U$-multiCat $(p)^{\frown} q$.

Let us consider $Y$, let $X$ be a subset of $Y$, and let $R$ be a total $Y$-defined binary relation. One can check that $R \uparrow X$ is total.

The following propositions are true:

(34) If $u=u_{1}$, then $\left(u_{1}, x_{2}\right)$-SymbolSubstIn $\langle u\rangle=\left\langle x_{2}\right\rangle$ and if $u \neq u_{1}$, then $\left(u_{1}, x_{2}\right)$-SymbolSubstIn $\langle u\rangle=\langle u\rangle$.

(35) If $u=u_{1}$, then $\left(u_{1}\right.$ SubstWith $\left.u_{2}\right)(\langle u\rangle)=\left\langle u_{2}\right\rangle$ and if $u \neq u_{1}$, then $\left(u_{1}\right.$ SubstWith $\left.u_{2}\right)(\langle u\rangle)=\langle u\rangle$.

(36) $(x$ SubstWith $u)\left(q_{1} \frown q_{2}\right)=(x \operatorname{SubstWith} u)\left(q_{1}\right)^{\frown}(x \operatorname{SubstWith} u)\left(q_{2}\right)$.

(37) If $p$ is $U^{*}$-valued, then $(x \operatorname{SubstWith} u)(U$-multiCat $(p))=U$-multiCat $((x \operatorname{SubstWith} u) \cdot p)$.

(38) (The concatenation of $U)^{\circ}\left(\operatorname{id}_{U^{1}}\right)=\{\langle u, u\rangle: u$ ranges over elements of $U\}$.

Let us consider $f, U, u$. One can verify that $(f\lceil U)(u)-f(u)$ is empty.

Let us consider $f, U_{1}, U_{2}$, let $u$ be an element of $U_{1}$, and let $g$ be a function from $U_{1}$ into $U_{2}$. Observe that $(f \cdot g)(u) \cdot f(g(u))$ is empty.

One can verify that every integer number which is non negative is also natural.

Let $x, y$ be real numbers. One can verify that $\max (x, y)-x$ is non negative.

The following proposition is true

(39) If $x$ is boolean, then $x=1$ iff $x \neq 0$.

Let us consider $Y$ and let $X$ be a subset of $Y$. Note that $X \backslash Y$ is empty.

Let us consider $x, y$. Observe that $\{x\} \backslash\{x, y\}$ is empty and $\langle x, y\rangle_{1} \dot{-} x$ is empty.

Let us consider $x, y$. Observe that $\langle x, y\rangle_{2} \dot{-} y$ is empty.

Let $n$ be a positive natural number and let $X$ be a non empty set. Note that there exists an element of $X^{*} \backslash\{\emptyset\}$ which is $n$-element.

Let us consider $m_{1}$. One can verify that every finite sequence which is $m_{1}+0$ element is also non empty.

Let us consider $R, x$. Note that $R$ null $x$ is relation-like.

Let $f$ be a function-like set and let us consider $x$. One can check that $f$ null $x$ is function-like. 
Let $p$ be a finite sequence-like binary relation and let us consider $x$. One can check that $p$ null $x$ is finite sequence-like.

Let us consider $p, x$. Observe that $p$ null $x$ is len $p$-element.

Let $p$ be a non empty finite sequence. Note that len $p$ is non zero.

Let $R$ be a binary relation and let $X$ be a set. Observe that $R \uparrow X$ is $X$-defined.

Let us consider $x$ and let $e$ be an empty set. Observe that $e$ null $x$ is empty.

Let us consider $X$ and let $e$ be an empty set. One can verify that $e$ null $X$ is $X$-valued.

Let $Y$ be a non empty finite sequence-membered set. One can check that every function which is $Y$-valued is also finite sequence-yielding.

Let us consider $X, Y$. Note that every element of $\left(Y^{*}\right)^{X}$ is finite sequenceyielding.

We now state the proposition

(40) If $f$ is $X^{*}$-valued, then $f(x) \in X^{*}$.

Let us consider $m, n$ and let $p$ be an $m$-element finite sequence. Observe that $p$ null $n$ is Seg $m+n$-defined.

Let us consider $m, n$, let $p$ be an $m$-element finite sequence, and let $q$ be an $n$-element finite sequence. Observe that $p^{\frown} q$ is $m+n$-element.

The following two propositions are true:

(41) Let $p_{1}, p_{2}, q_{1}, q_{2}$ be finite sequences. Suppose $p_{1}$ is $m$-element but $q_{1}$ is $m$-element but $p_{1} \frown p_{2}=q_{1} \frown q_{2}$ or $p_{2} \frown p_{1}=q_{2} \frown q_{1}$. Then $p_{1}=q_{1}$ and $p_{2}=q_{2}$.

(42) If $U$-multiCat $(x)$ is $U_{1}$-valued and $x \in\left(U^{*}\right)^{*}$, then $x$ is a finite sequence of elements of $U_{1}{ }^{*}$.

Let us consider $U$. One can verify that there exists a reflexive binary relation on $U$ which is total.

Let us consider $m$. Note that every finite sequence which is $m+1$-element is also non empty.

Let us consider $U, u$. Note that $\operatorname{id}_{U}(u) \dot{-} u$ is empty.

Let us consider $U$ and let $p$ be a $U$-valued non empty finite sequence. Observe that $\{p(1)\} \backslash U$ is empty.

Next we state the proposition

(43) If $x_{1}=x_{2}$, then $f+\cdot\left(x_{1} \longmapsto y_{1}\right)+\cdot\left(x_{2} \longmapsto y_{2}\right)=f+\cdot\left(x_{2} \longmapsto y_{2}\right)$ and if $x_{1} \neq$ $x_{2}$, then $f+\cdot\left(x_{1} \longmapsto y_{1}\right)+\cdot\left(x_{2} \longmapsto y_{2}\right)=f+\cdot\left(x_{2} \longmapsto y_{2}\right)+\cdot\left(x_{1} \longmapsto y_{1}\right)$.

Let us consider $X, U$. Note that there exists an $X$-defined function which is $U$-valued and total.

Let us consider $X, U$, let $P$ be a $U$-valued total $X$-defined binary relation, and let $Q$ be a total $U$-defined binary relation. One can verify that $P \cdot Q$ is total.

We now state the proposition

(44) If $p^{\frown} p_{1} \frown p_{2}$ is $X$-valued, then $p_{2}$ is $X$-valued and $p_{1}$ is $X$-valued and $p$ is $X$-valued. 
Let us consider $X$ and let $R$ be a binary relation. One can check that $R$ null $X$ is $X \cup \operatorname{rng} R$-valued.

Let $X, Y$ be functional sets. One can verify that $X \cup Y$ is functional.

Let us note that every set which is finite sequence-membered is also finitemembered.

Let $X$ be a functional set. The functor SymbolsOf $X$ is defined by:

(Def. 24) SymbolsOf $X=\bigcup\{\operatorname{rng} x ; x$ ranges over elements of $X \cup\{\emptyset\}: x \in X\}$.

Let us observe that there exists a set which is trivial, finite sequencemembered, and non empty.

Let $X$ be a functional finite finite-membered set. Note that SymbolsOf $X$ is finite.

Let $X$ be a finite finite sequence-membered set. One can verify that SymbolsOf $X$ is finite.

The following proposition is true

(45) SymbolsOf $\{f\}=\operatorname{rng} f$.

Let $z$ be a non zero complex number. One can check that $|z|$ is positive.

The scheme $S c 1$ deals with a set $\mathcal{A}$, a set $\mathcal{B}$, and a unary functor $\mathcal{F}$ yielding a set, and states that:

$\{\mathcal{F}(x) ; x$ ranges over elements of $\mathcal{A}: x \in \mathcal{A}\}=\{\mathcal{F}(x) ; x$ ranges over elements of $\mathcal{B}: x \in \mathcal{A}\}$

provided the following condition is satisfied:

- $\mathcal{A} \subseteq \mathcal{B}$.

Let $X$ be a functional set. Then SymbolsOf $X$ can be characterized by the condition:

(Def. 25) SymbolsOf $X=\bigcup\{\operatorname{rng} x ; x$ ranges over elements of $X: x \in X\}$.

One can prove the following propositions:

(46) For every functional set $B$ and for every subset $A$ of $B$ holds SymbolsOf $A \subseteq$ SymbolsOf $B$.

(47) For all functional sets $A, B$ holds SymbolsOf $(A \cup B)=$ SymbolsOf $A \cup$ SymbolsOf $B$.

Let us consider $X$ and let $F$ be a subset of $2^{X}$. One can verify that $\bigcup F \backslash X$ is empty.

The following four propositions are true:

(48) $X=(X \backslash Y) \cup X \cap Y$.

(49) If $A^{m}$ meets $B^{n}$, then $m=n$.

(50) If $B$ is $D$-prefix and $A \subseteq B$, then $A$ is $D$-prefix.

(51) $f \subseteq g$ iff for every $x$ such that $x \in \operatorname{dom} f$ holds $x \in \operatorname{dom} g$ and $f(x)=$ $g(x)$.

Let us consider $U$. One can verify that every element of $\left(U^{*} \backslash\{\emptyset\}\right)^{*}$ which is non empty is also non empty yielding. 
Let $e$ be an empty set. One can verify that every element of $e^{*}$ is empty.

The following proposition is true

(52)(i) If $U_{1}$-multiCat $(x) \neq \emptyset$ and $U_{2}$-multiCat $(x) \neq \emptyset$, then $U_{1}$-multiCat $(x)=U_{2}$-multiCat $(x)$,

(ii) if $p$ is $\emptyset^{*}$-valued, then $U_{1}$-multiCat $(p)=\emptyset$, and

(iii) if $U_{1}$-multiCat $(p)=\emptyset$ and $p$ is $U_{1}{ }^{*}$-valued, then $p$ is $\emptyset^{*}$-valued.

Let us consider $U, x$. Note that $U$-multiCat $(x)$ is $U$-valued.

Let us consider $x$. The functor $x$ null is defined by:

(Def. 26) $\quad x$ null $=x$.

Let $Y$ be a set with non empty elements. Observe that every $Y$-valued binary relation which is non empty is also non empty yielding.

Let us consider $X$. Observe that $X \backslash\{\emptyset\}$ has non empty elements.

Let $X$ be a set with non empty elements. One can check that every subset of $X$ has non empty elements.

Let us consider $U$. Note that $U^{*}$ is infinite. Observe that $U^{*}$ has a non-empty element.

Let $X$ be a set with a non-empty element. Note that there exists a subset of $X$ which is non empty and has non empty elements.

One can prove the following propositions:

(53) If $U_{1} \subseteq U_{2}$ and $Y \subseteq U_{1}{ }^{*}$ and $p$ is $Y$-valued and $p \neq \emptyset$ and $Y$ has non empty elements, then $U_{1}$-multiCat $(p)=U_{2}$-multiCat $(p)$.

(54) If there exists $p$ such that $x=p$ and $p$ is $X^{*}$-valued, then $U$-multiCat $(x)$ is $X$-valued.

Let us consider $X, m$. Observe that $X^{m} \backslash X^{*}$ is empty.

The following two propositions are true:

(55) $\quad(A \cap B)^{*}=A^{*} \cap B^{*}$.

(56) $(P \cup Q) \uparrow X=P \uparrow X \cup Q \uparrow X$.

Let us consider $X$. One can check that $2^{X} \backslash X$ is non empty.

Let us consider $X$ and let $R$ be a binary relation. One can verify that $R$ null $X$ is $X \cup \operatorname{dom} R$-defined.

Next we state the proposition

(57) $f\lceil X+\cdot g=f \uparrow(X \backslash \operatorname{dom} g) \cup g$.

We now state the proposition

(58) If $y \notin \pi_{2}(X)$, then $A \times\{y\}$ misses $X$.

Let us consider $X$. The functor $X$-freeCountableSet is defined by:

(Def. 27) $X$-freeCountableSet $=\mathbb{N} \times\left\{\right.$ the element of $\left.2^{\pi_{2}(X)} \backslash \pi_{2}(X)\right\}$.

Next we state the proposition

(59) $X$-freeCountableSet $\cap X=\emptyset$ and $X$-freeCountableSet is infinite. 
Let us consider $X$. Observe that $X$-freeCountableSet is infinite. Observe that $X$-freeCountableSet $\cap X$ is empty. One can verify that $X$-freeCountableSet is countable.

One can check that $\mathbb{N} \backslash \mathbb{Z}$ is empty.

Let us consider $x, p$. Observe that $(\langle x\rangle \frown p)(1)-x$ is empty.

Let us consider $m$, let $m_{0}$ be a zero number, and let $p$ be an $m$-element finite sequence. Note that $p$ null $m_{0}$ is total.

Let us consider $U, q_{1}, q_{2}$. One can check that $U$-multiCat $\left(\left\langle q_{1}, q_{2}\right\rangle\right) \dot{-} q_{1} \frown q_{2}$ is empty.

\section{REFERENCES}

[1] Broderick Arneson and Piotr Rudnicki. Recognizing chordal graphs: Lex BFS and MCS. Formalized Mathematics, 14(4):187-205, 2006, doi:10.2478/v10037-006-0022-z.

[2] Grzegorz Bancerek. Cardinal numbers. Formalized Mathematics, 1(2):377-382, 1990.

[3] Grzegorz Bancerek. The fundamental properties of natural numbers. Formalized Mathematics, 1(1):41-46, 1990.

[4] Grzegorz Bancerek. König's theorem. Formalized Mathematics, 1(3):589-593, 1990.

[5] Grzegorz Bancerek. The ordinal numbers. Formalized Mathematics, 1(1):91-96, 1990.

[6] Grzegorz Bancerek. Monoids. Formalized Mathematics, 3(2):213-225, 1992.

[7] Grzegorz Bancerek and Krzysztof Hryniewiecki. Segments of natural numbers and finite sequences. Formalized Mathematics, 1(1):107-114, 1990.

[8] Grzegorz Bancerek and Yasunari Shidama. Introduction to matroids. Formalized Mathematics, 16(4):325-332, 2008, doi:10.2478/v10037-008-0040-0.

[9] Grzegorz Bancerek and Andrzej Trybulec. Miscellaneous facts about functions. Formalized Mathematics, 5(4):485-492, 1996.

[10] Czesław Byliński. Basic functions and operations on functions. Formalized Mathematics, $1(\mathbf{1}): 245-254,1990$.

[11] Czesław Byliński. Binary operations. Formalized Mathematics, 1(1):175-180, 1990.

[12] Czesław Byliński. The complex numbers. Formalized Mathematics, 1(3):507-513, 1990.

[13] Czesław Byliński. Finite sequences and tuples of elements of a non-empty sets. Formalized Mathematics, 1(3):529-536, 1990.

[14] Czesław Byliński. Functions and their basic properties. Formalized Mathematics, 1(1):55$65,1990$.

[15] Czesław Byliński. Functions from a set to a set. Formalized Mathematics, 1(1):153-164, 1990.

[16] Czesław Byliński. The modification of a function by a function and the iteration of the composition of a function. Formalized Mathematics, 1(3):521-527, 1990.

[17] Czesław Byliński. Partial functions. Formalized Mathematics, 1(2):357-367, 1990.

[18] Czesław Byliński. Some basic properties of sets. Formalized Mathematics, 1(1):47-53, 1990.

[19] Agata Darmochwał. Finite sets. Formalized Mathematics, 1(1):165-167, 1990.

[20] M. Lothaire. Algebraic combinatorics on words. Cambridge Univ Pr, 2002.

[21] Beata Padlewska. Families of sets. Formalized Mathematics, 1(1):147-152, 1990.

[22] Andrzej Trybulec. Binary operations applied to functions. Formalized Mathematics, 1(2):329-334, 1990.

[23] Andrzej Trybulec. Domains and their Cartesian products. Formalized Mathematics, 1(1):115-122, 1990.

[24] Andrzej Trybulec. Tuples, projections and Cartesian products. Formalized Mathematics, $1(\mathbf{1}): 97-105,1990$.

[25] Michał J. Trybulec. Integers. Formalized Mathematics, 1(3):501-505, 1990.

[26] Zinaida Trybulec. Properties of subsets. Formalized Mathematics, 1(1):67-71, 1990.

[27] Edmund Woronowicz. Many-argument relations. Formalized Mathematics, 1(4):733-737, 1990.

[28] Edmund Woronowicz. Relations and their basic properties. Formalized Mathematics, 1(1):73-83, 1990. 
[29] Edmund Woronowicz. Relations defined on sets. Formalized Mathematics, 1(1):181-186, 1990.

[30] Edmund Woronowicz and Anna Zalewska. Properties of binary relations. Formalized Mathematics, 1(1):85-89, 1990.

Received December 29, 2010 